\title{
Flutter Characterstics of a Flexible Filament and its Control
}

\author{
Kamal Poddar, Ashwini N. Arekar, Dhiraj Kumar
}

\begin{abstract}
Flow induced flutter of a thin flexible filament attached to the trailing-edge of a NACA 0015 airfoil was studied experimentally in a low-speed wind tunnel. Hotwire and PIV measurements were carried out to study the nature of the flow field around the airfoil and the filament at different wind speeds. At a critical wind speed, the filament starts to flutter and achieves a mode of limit cycle oscillation (LCO). Beyond the critical wind speed, the flutter frequency of the filament increases with the increase in wind speed. Also, a change in flutter mode, from $2^{\text {nd }}$ to $3^{\text {rd }}$ mode LCO, was observed at a higher wind speed. A trip wire was introduced to the fore-body to control the flutter characteristics of the filament by modifying the boundary layer characteristics. Trip wire was attached to the airfoil surface (one or both sides of the airfoil) at different chord-wise locations. A delay in the mode shape transition from 2nd mode to 3rd mode LCO was observed due to the presence of the trip wire at certain locations. Trip wires attached to both sides of the airfoil, at a distance of $30 \%$ of the chord from the leading edge, was found to be the most effective in delaying the transition.
\end{abstract}

Keywords - Flexible filament; Flow-induced flutter, Limit cycle oscillation; Flow control

\section{Introduction}

The flow-induced flutter of a filament is a classic example of fluid structure interaction where a moving fluid interacts with a movable or deformable structure. The interaction between a flexible structure and the surrounding fluid gives rise to a variety of phenomena such as, wind-induced vibration of an aircraft wing and its control surfaces, vibration of wind turbine blades and flutter of a flag in a moving fluid. The movement of the fluid can induce a motion or deformation to the solid body which in turn feeds back and alters the flow field around it. The flow-induced motion of a rigid body such as, flutter of a hinged plate in a uniform stream has finite degrees of freedom [1]. In contrast, the motion of a flexible filament, fixed at its one end, has infinite degrees of freedom. The mutual interaction between the fluid and the flexible structure is quite complex in nature. The study of flutter of a flexible filament has a relevance to several real life phenomena such as, fluttering of a flag, paper flutter induced by high speed operations of a printing machine [2], human snoring [3], and locomotion of fish [4]. Understanding the flutter characteristics of a flexible filament is also important for exploring its physical applications such as, autonomous vehicle propulsion, aeroelastic energy harvesters [5] and ocean power generator using flexible piezoelectric sheets [6].

The flutter of a flexible plate in a uniform flow can originate due to flow disturbances presence in the free-stream or induced in the wake of mast [7]. In experimental study [8],

Kamal Poddar, Ashwini N. Arekar, Dhiraj Kumar

Department of Aerospace Engineering

IIT Kanpur, India a hysteresis has been observed for a high aspect ratio plate which is attributed to the span-wise deformation in the plate. Depending on the aspect ratio and the flow velocity, a flexible filament can have different modes of flutter such as, single neck (mode I) and double neck (mode II) [2,9].

In the previous investigations, the initiation and various modes of flutter have been studied. Many predictions have been made regarding the initiation of flutter and the hysteresis. However, the cause of flutter initiation and the hysteresis is not yet well understood. Also, the change in mode of flutter is not well explored. In the present study, flutter initiation and mode shape transition have been investigated. An attempt was also made to control the flutter characteristics of the filament by modifying the boundary layer characteristics over the forebody.

\section{Methodology}

In this section, experimental setup, measurement techniques, data acquisition system and analysis methodologies used in the present study are discussed.

\section{A. Experimental Setup}

The experiments were performed in an open circuit, low speed wind tunnel at low speed aerodynamics laboratory, IIT Kanpur. The cross-section of the wind tunnel is $0.4 \mathrm{~m} \times 0.5 \mathrm{~m}$ and the experiments were carried out in the wind speed range $1-20 \mathrm{~m} / \mathrm{s}$.

The wind-tunnel tests were carried out on a symmetric airfoil (NACA 0015) with a flexible filament attached to its trailing edge. The airfoil was made of acrylic and the flexible filament was cut from a transparent polyester (polyethylene terephthalate) sheet of thickness $\left(t_{f}\right) 125$ microns. The chord (C) and the span $(\mathrm{H})$ of the airfoil were $120 \mathrm{~mm}$ and $400 \mathrm{~mm}$, respectively. The chord-wise length $(l)$ and the span-wise length $(h)$ of the filament were kept constant $(l=100 \mathrm{~mm}$ and $h=120 \mathrm{~mm}$ ) for the present study. The rear part of the airfoil was split into two halves and the filament was clamped between them as shown in figure 1.

Velocity fluctuations along the sides of the airfoil and the filament, and in the down-stream wake, were detected using four single-wire hotwire probes. A schematic diagram of the hotwire measurement setup is shown in figure 2. Multichannel CTA system (54N81, Dantec Dynamics) was used for the hotwire measurement and the signals were sampled at a rate of 5000 samples per second using National Instruments data acquisition system (NI PXIe-1073 PXI chassis with PXIe6281 DAQ module). Hardware filter setting of the multi CTA system was set at $1 \mathrm{kHz}$ low-pass filter, for all the four probes. To obtain the frequencies of the flow fluctuation, the time domain hotwire signals were transformed into frequency domain using FFT (Fast Fourier Transformation). 


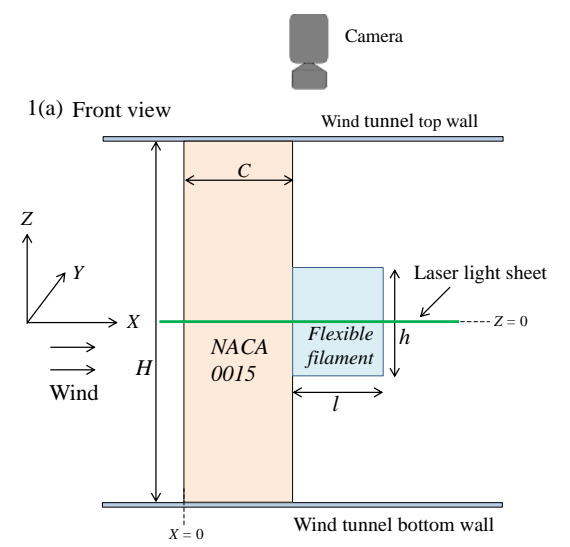

1(b) Top view

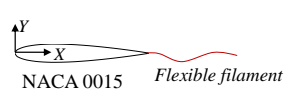

1(c) Airfoil filament attachment

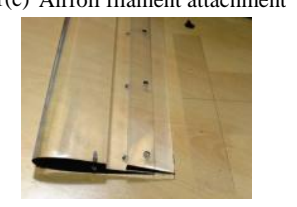

Figure 1. Experimental setup: a) Front view, b) Top view and c) airfoilfilament attachment.

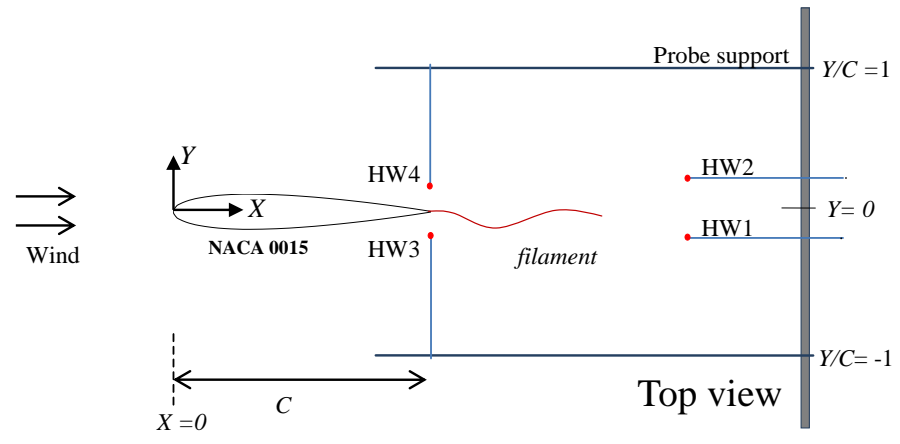

Figure 2. A schematic of the experimental setup.

Positions of the hotwire probes were chosen as per model configurations and test requirements. In most of the cases, the upstream hotwires (HW3 \& HW4) were placed, symmetrically on both sides of the airfoil, near the trailing edge $(X / C=1)$ of the airfoil. The downstream probes (HW1 \& HW2) were placed in the wake of the airfoil or in the wake of the filament. The flutter frequency of the filament was obtained from the power spectrum of the downstream hotwire data as the movement of the filament induces a large amplitude velocity fluctuation in the wake.

2D PIV measurements were carried out to study the flow field around the airfoil with and without the filament. Measurement plane at mid-span of the airfoil was illuminated with a dual pulse laser (Quantel, 200mJ) light sheet and the images were captured using an 8MP CCD camera (Imperx, B3340M) as shown in figure 1(a). Velocity field from the PIV data was obtained by processing the images in IDT Provision software. The velocity field was normalized with the freestream velocity and the spatial dimensions (X, Y) were normalized with the chord of the airfoil.

A DSLR camera (Nikon D750) was used to capture the motion of the filament and the images were further processed to obtain the flutter amplitude. Mid span of the filament was illuminated using a horizontal laser light sheet flashing at a high frequency and time interval snaps of the illuminated filament was captured using the DSLR camera.

\section{B. Filament characterization}

Physical properties of the filament play a key role in its flutter characteristics. Therefore, the physical properties such as, density and Young's modulus of elasticity were obtained experimentally. Density was calculated by measuring the weight of the filament. The modulus of elasticity was obtained by measuring the tip deflection (bending) of cantilevered filament (clamped horizontally) due to self-weight and equating it with the theoretical deflection of a cantilevered beam under uniformly distributed load. For the flexible filament used in present study, measured value of the density is $1350 \mathrm{~kg} / \mathrm{m}^{3}$ and the modulus of elasticity is $4 \mathrm{GPa}$, approximately.

Using the experimentally obtained material properties, the natural frequencies of the filament for the different modes of free vibration [10] were estimated as follows:

$$
\begin{aligned}
& \text { 1st mode }=\sim 3.5 \mathrm{~Hz} \\
& \text { 2nd mode }=\sim 21 \mathrm{~Hz}
\end{aligned}
$$

\section{RESULTS AND DISCUSSION}

The behaviour of the flexible filament at different Reynolds number $(7,000$ to 140,000$)$, from its stable state to different modes of limit cycle oscillation is discussed in this section. The effect of the trip wire attached to airfoil surface on the flow field around it and on the flutter characteristics of the filament is discussed.

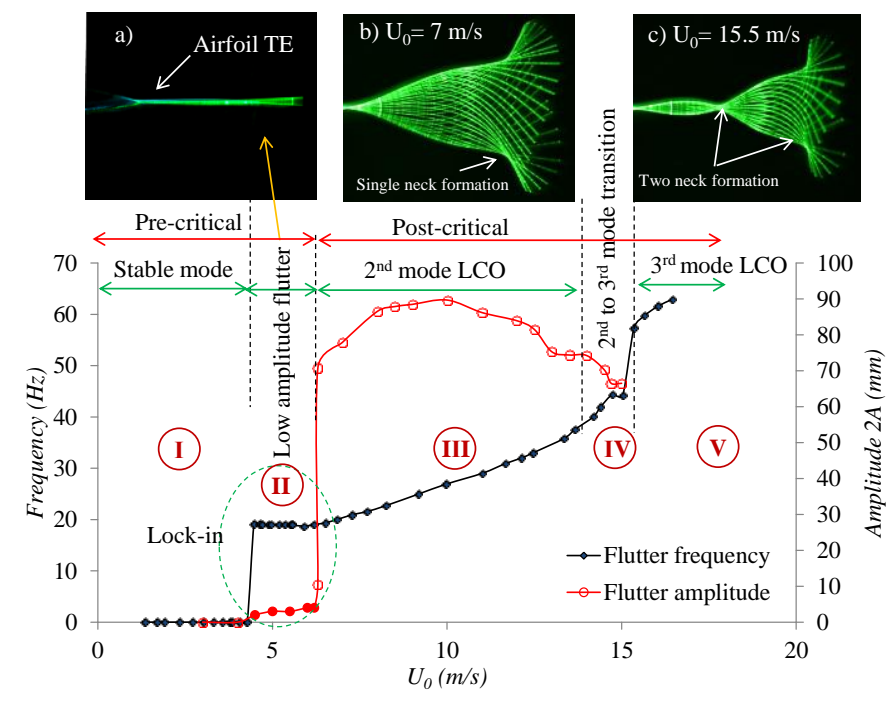

Figure 3. Different regimes of flutter of a filament.

On gradually increasing the wind speed, the filament attached to the airfoil starts to flutter at a certain wind speed (referred as critical wind speed). The study of the flutter phenomenon can be broadly classified into two regimes: a) Pre-critical regime, b) Post-critical regime. Behavior of the filament in various regimes is shown in figure 3 . The figure shows the flutter frequency and amplitude at different wind speeds. At a lower wind speed the filament remains in a stable 
mode aligned with the flow. By further increasing the wind speed, a low-amplitude flutter regime is observed as shown in figure 3. In this regime, the frequency of the low amplitude flutter remains nearly constant with the increasing wind speed. At the critical wind speed, the amplitude of flutter increases rapidly and the filament achieves a mode of limit cycle oscillation (LCO). The mode shapes of the filament in different regimes are shown in figures $3(\mathrm{a}-\mathrm{c})$.

\section{A. Pre-critical regime}

Pre-critical regime can be subdivided into stable mode regime and low-amplitude flutter regime. In stable mode, the filament remains stationary and aligned with the axial wind. In the present study, this regime is classified as stable mode regime (regime I). Whereas, in the low amplitude flutter regime, the filament is found to have an intermittent flutter of very low amplitude. The figures 4 (a \& c) show the signals of the hotwires (HW3 \& HW4) positioned near the trailing edge of the airfoil without filament and the hotwires (HW3 \& HW4) signal for airfoil with filament in the stable mode regime, respectively. The hotwire signals for airfoil without the filament are out of phase (figure 3a); whereas, the signal of the probes positioned near the trailing edge of the airfoil with filament are almost in phase as shown in figure 3(c). Therefore, it can be inferred that the vortices, shed behind the airfoil without filament, are out of phase. In contrast the vortices shed from the airfoil with filament are almost inphase.
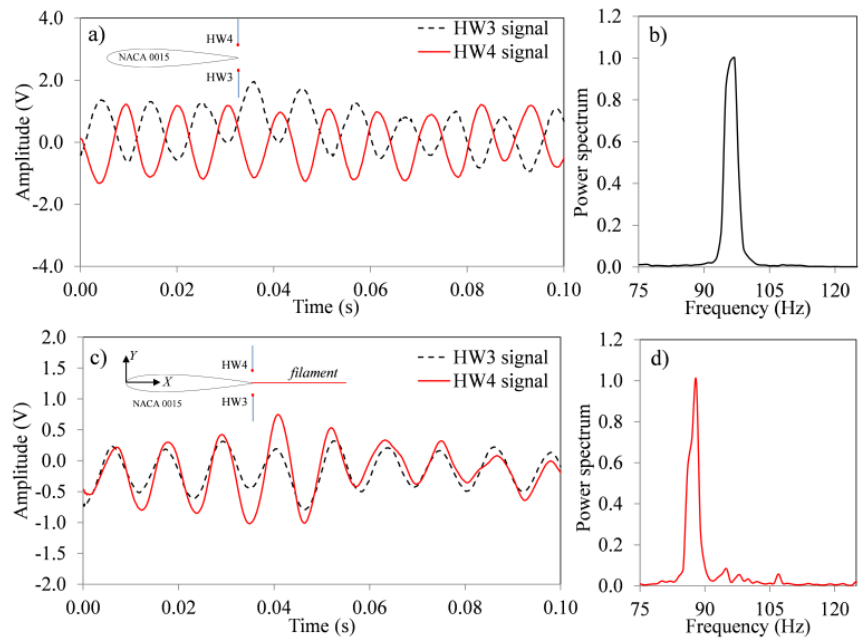

Figure 4: Hotwire signals (conditioned) and power spectra: a) \& b) for airfoil alone; c) \& d) for airfoil with filament in stable regime at $R e=30,000$.

The power spectra of the hotwire signals for the airfoil without and with filament are shown in figures $4 \mathrm{~b} \& 4 \mathrm{~d}$, respectively. It can be noticed, from these figures, that the vortex shedding frequency for airfoil with filament is significantly lower than the shedding frequency for the airfoil alone. The difference in the shedding frequencies was observed for a wide range of Reynolds number in the stable regime.
Figure 5 shows the instantaneous along-wind velocity contours obtained from the PIV measurements carried out in the stable mode (regime I). The presence of small scale vortices can be observed on both sides of the filament attached to the airfoil in figure 5 . As detected by the hotwire probes (figure 4) the vortices along the both sides of the filament appear to be in-phase.

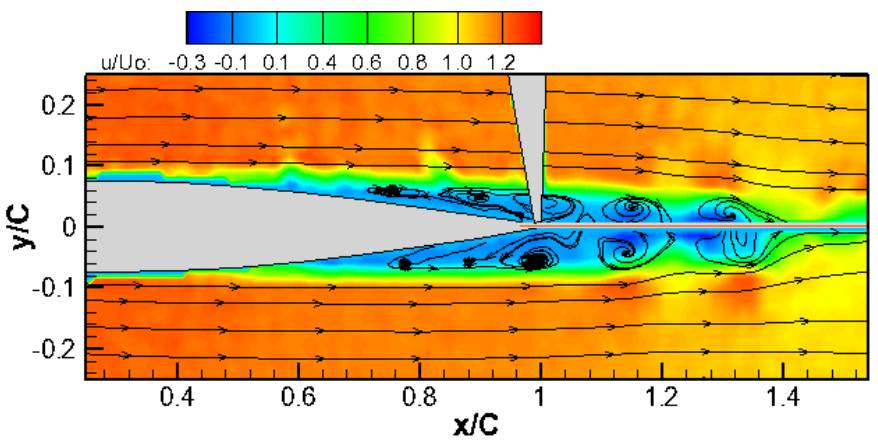

Figure 5. Instantaneous along-wind velocity contour for airfoil with filament at $\mathrm{Re}=21,000$.

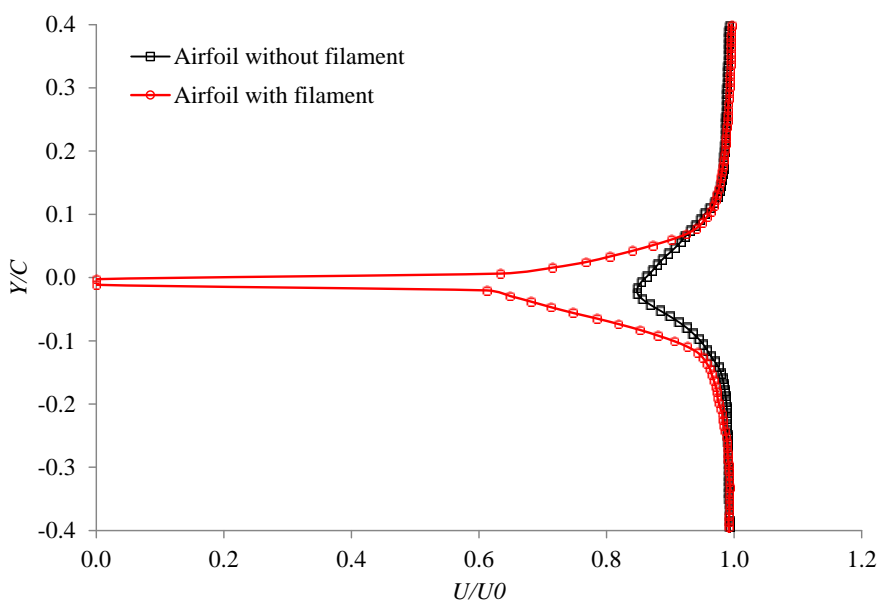

Figure 6. Wake profiles for airfoil alone and airfoil with filament, at $\mathrm{X} / \mathrm{C}=1.5$, in stable mode regime at $\mathrm{Re}=28,000$.

The wake profiles for the airfoil with and without filament, at $X / C=1.5$ in the stable mode regime at $R e=28,000$, are shown in figure 6 . The modification in the wake profile of the airfoil due to the filament can be easily seen in the figure. The higher velocity deficit for an airfoil with filament is mainly due to the skin friction loss over the filament surface. It can also be observed that the velocity is close to zero at the center of the wake profile for the airfoil with filament, which is due to no slip condition over the filament surface.

Low-amplitude flutter regime is characterized by a low amplitude intermittent oscillation of the filament. The mode shape of the filament in this regime is shown in figure 3(a). In this regime, the flutter frequency of the filament is almost constant $(\sim 19 \mathrm{~Hz})$. It was observed that the frequency of lowamplitude flutter is close to the estimated $2^{\text {nd }}$ mode natural frequency $(\sim 21 \mathrm{~Hz})$ of the filament as discussed in section II B. Therefore, it can be inferred that the low-amplitude flutter of 
the filament is locked to the 2nd mode natural frequency of the filament.

\section{B. Post-critical regime}

At the critical wind speed, the amplitude of flutter rapidly increases and the mode of the filament oscillation changes from the low-amplitude flutter to a high amplitude limit cycle oscillation. The mode shapes of the filament in the post critical regime are shown in figures $3(\mathrm{~b} \& \mathrm{c})$. At the critical wind speed, the high amplitude LCO begins with the flutter frequency of $19 \mathrm{~Hz}$ which is close to the $2^{\text {nd }}$ mode natural frequency of the filament. Also, the curvature of the filament, as shown in figure 3(b), is similar to the curvature of a cantilever beam in its $2^{\text {nd }}$ mode free vibration. Therefore, it can be said that the large amplitude flutter begins with the $2^{\text {nd }}$ mode LCO. A single neck formation can be observed in the mode shape image of the filament (Figure $3 \mathrm{~b}$ ) in $2^{\text {nd }}$ mode LCO. In this regime, the amplitude of flutter increases initially with speed and then decreases with further increase in wind speed. However, the flutter frequency increases continuously with increasing wind speed.

In the range of wind speed $13 \mathrm{~m} / \mathrm{s}$ to $15.5 \mathrm{~m} / \mathrm{s}$ (regime IV as shown in figure 3 ), the filament is found to have an unstable mode of oscillation. In this speed range, flutter frequency and amplitude of the filament change with time. At the wind speed close to $15.5 \mathrm{~m} / \mathrm{s}$, the filament again attains a stable mode of limit cycle oscillation. In this regime (regime V), the curvature or the mode shape of the filament, as shown in figure 3c, is similar to the curvature of a cantilever beam in its 3rd mode of free vibration. The formation of two necks can be noticed in the mode shape of the filament (figure 3c). This regime (regime V) can be referred as $3^{\text {rd }}$ mode LCO. From the above discussion, it can be said that the limit cycle oscillation of the filament initiates with the $2^{\text {nd }}$ mode of vibration and goes into $3^{\text {rd }}$ mode of vibration at a higher wind speed.

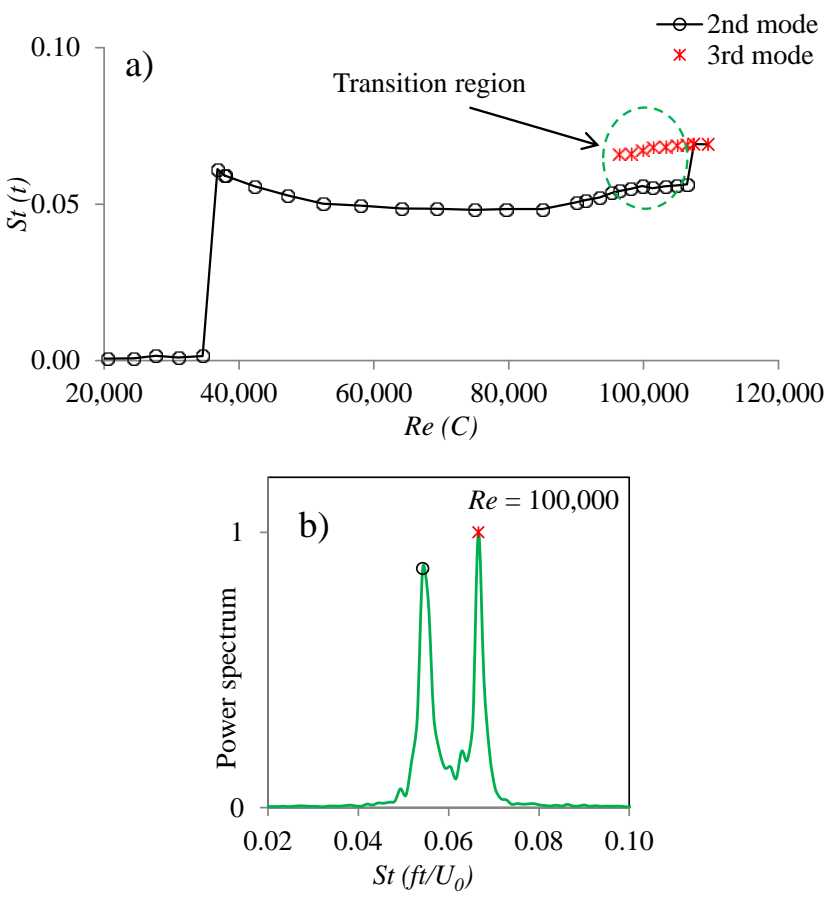

Figure 7. a) Non-dimensional flutter frequency at different Reynolds number; b) Hotwire power spectrum in transition region at $R e=100,000$

Figure 7a shows the non-dimensional flutter frequencies of the filament (Strouhal number, $S t=f t / U$ where, $f$ is the flutter frequency), obtained from the hotwire measurements, at different Reynolds numbers. During the transition from $2^{\text {nd }}$ mode to $3^{\text {rd }}$ mode LCO ( $R e=95,000$ to 105,000$)$, multiple frequencies were detected by the hotwires located in the wake of the filament. Power spectrum of the hotwire signal at $R e=$ 100,000, in the transition regime, is shown in Figure 7b where, two different frequency peaks $(42 \mathrm{~Hz} \mathrm{\&} 51 \mathrm{~Hz})$ can be observed. The power spectrum shown in Figure $7 \mathrm{~b}$ is corresponding to 30 seconds of hotwire data. The two significant frequency peaks $(42 \mathrm{~Hz} \& 51 \mathrm{~Hz}$ at $R e=100,000)$ in the power spectrum indicates that filament flutters at $42 \mathrm{~Hz}$ for a period of time and at $51 \mathrm{~Hz}$ for another period of time, at a given wind speed. The Strouhal number corresponding to both the frequency peaks, in the transition region, are shown in figure $7 \mathrm{a}$. Also, during the transition, the mode shape of the filament was found switching intermittently between singleneck and double neck modes.

\section{Control of flutter characteristics}

The flow structure around the filament plays a key role in governing its flutter characteristics. Therefore, the flow over the fore-body (airfoil) and its wake are expected to have a significant impact on the flutter of the filament. In the present study, an attempt was made to control the flutter characteristics by modifying the boundary layer over the forebody. In order to modify the boundary layer characteristics over the airfoil, trip wires were used and their effect on flutter characteristics of the filament is discussed in this section.

Figure 8 shows the effect of a trip wire, attached to one side of the airfoil at different chord-wise locations, on the flow 
field around the model in the stable mode regime. The asymmetry in the mean flow field due the presence of the trip wire on one side of the airfoil can be easily observed in figures 8(a \& c) for $X / C=0.3 \& 0.4$, respectively. However, the effect of the trip wire at $X / C=0.5$ is negligible (figure $8 \mathrm{e}$ ) as compared to that at $X / C=0.3 \& 0.4$. In figure 8a, it can be noticed that the flow over trip wire side reattaches to filament surface earlier (at lower $X / C$ ) than the flow over the other side of the filament. This is due to the fact that the trip wire enhances or promotes the turbulent nature of the flow. Similar observations can also be made in the respective instantaneous flow field shown in figures 8(b, d \& f). From figure 8, it can be inferred that the trip wire at $X / C=0.3$ has more effect over the flow field around the filament as compared to that at $X / C=$ $0.4 \& 0.5$.

The flow field around the airfoil with the trip wire at $X / C=$ 0.5 appears similar to the flow field without the trip wire. The effectiveness of a roughness device depends on its size with respect to the thickness of local boundary layer and the Reynolds number of the flow. Since, there is an adverse pressure gradient over the airfoil at $X / C=0.5$, the local boundary layer thickness is much higher than the diameter of the trip wires $(0.5 \mathrm{~mm})$ used in the present study. Therefore, the trip wires attached at $X / C=0.5$ are not effective in modifying the boundary layer.

\section{Instantaneous velocity contour}
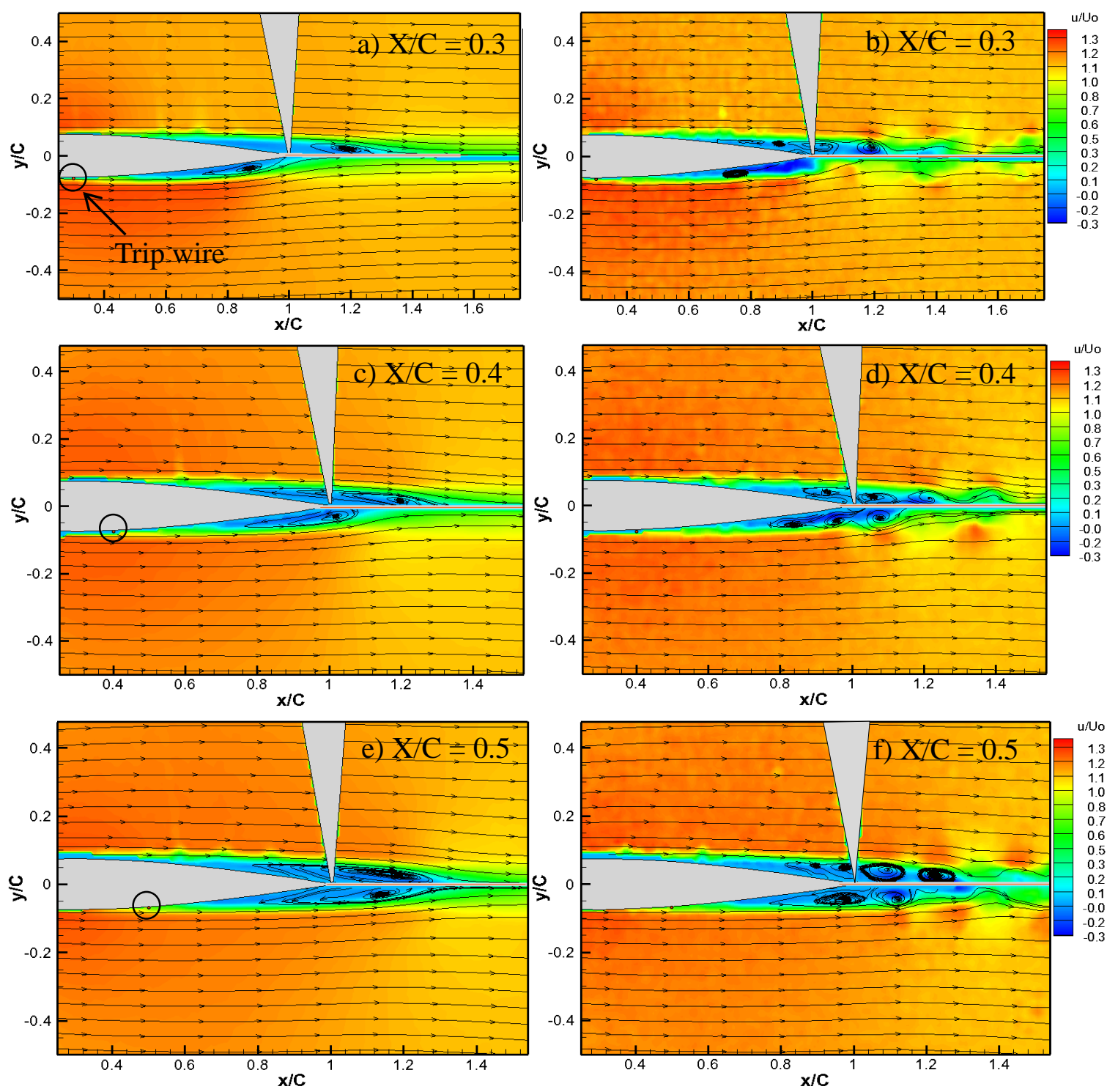

Figure 8. Effect of trip wire attached to one side of the airfoil at $X / C=0.3,0.4$ and 0.5 in stable mode regime at $R e=21,000$. 
Proc. of the Seventh Intl. Conf. on Advances in Civil, Structural and Mechanical Engineering - CSM 2018

Copyright (C) Institute of Research Engineers and Doctors, USA .All rights reserved.

ISBN: 978-1-63248-163-4 DOI: 10.15224/978-1-63248-163-4-16
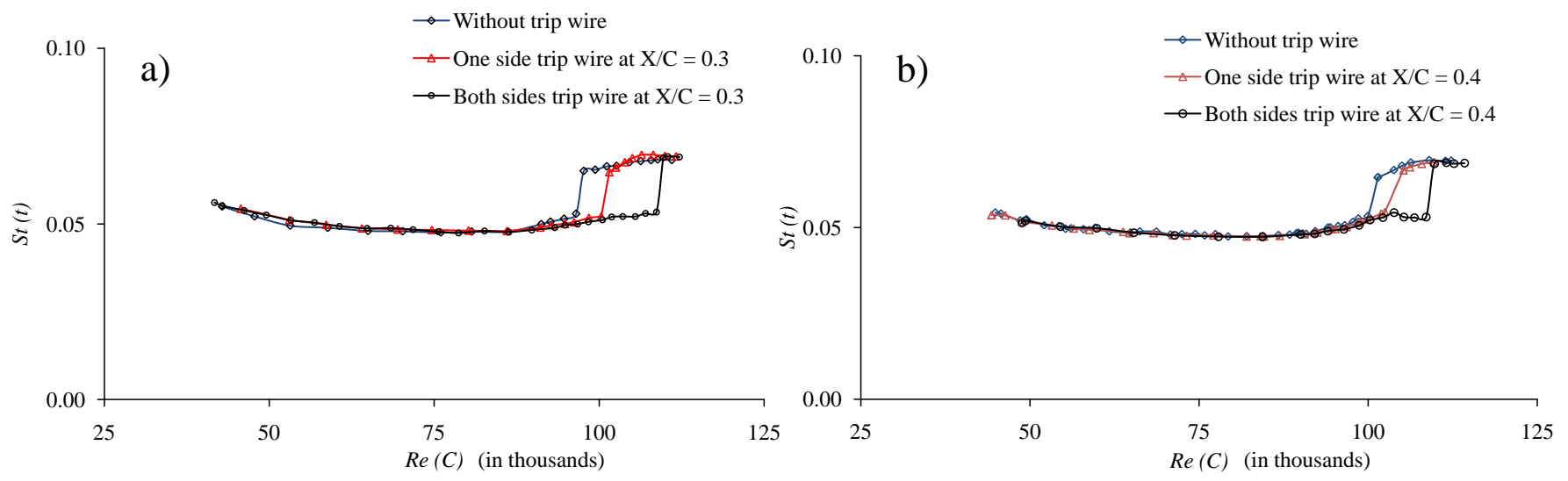

Figure 9. Effect of trip wire on change in mode from $2^{\text {nd }}$ to $3^{\text {rd }}$ mode.

The effect of the trip wire on the flutter frequency was studied for two different configurations. In one configuration, the trip wire was attached to only one side of the airfoil at a particular $X / C$ location. In another configuration, the trip wire was attached to both sides of the airfoil. Figure 9a shows the effect of trip wire, attached at $X / C=0.3$, on the flutter frequency of the filament. It can be observed that due to the presence of trip wire, the mode change from $2^{\text {nd }}$ mode to $3^{\text {rd }}$ mode LCO has been delayed significantly as compared to that of the filament attached to the airfoil without trip wire. It can also be noticed that trip wires attached to both sides of the airfoil surface, are more effective in delaying the mode change compared to the trip wire attached to only one side of the airfoil surface. Similar observations were also made for the trip wires attached at $X / C=0.4$, as shown in figure 9b. The reason behind the delay in mode change is not yet clearly understood and further investigation is being carried out.

\section{Conclusions}

The presence of the filament significantly modifies the flow field around the airfoil. PIV measurements show the presence of small scale vortices moving along the two sides of the filament at lower Reynolds number $(\mathrm{Re}=21,000)$. In the stable mode regime, the vortices shed along the alternate sides of the filament were found to be mostly in-phase unlike the alternate shedding behind the airfoil without the filament. Also, the vortex shedding frequencies for airfoil with filament was found to be significantly lower than the shedding frequency for the airfoil alone. At wind speed lower than the critical wind speed, there exists a low amplitude flutter regime in which the flutter of the filament is locked to a frequency close to the $2^{\text {nd }}$ mode natural frequency of the filament. At the critical wind speed, the flutter amplitude of the filament increases rapidly and the filament achieves a 2nd mode limit cycle oscillation. By further increasing the wind speed, the mode of flutter changes from $2^{\text {nd }}$ to 3rd mode LCO.

A delay in the mode shape transition from $2^{\text {nd }}$ mode to $3^{\text {rd }}$ mode LCO was observed for trip wire locations of $30 \%$ and $40 \%$ of the chord. The trip wires attached to both sides of the airfoil, at $30 \%$ of the chord, are found be the most effective in delaying this transition.

\section{Acknowledgment}

Assistance received from technical staffs of low speed aerodynamics lab is gratefully acknowledged.

\section{References}

[1] D. Kumar, K. Poddar, \& S. Kumar, (2018). Flow-induced oscillation of a rigid rectangular plate hinged at its leading edge. Physics of Fluids, 30(6), 063601.

[2] Y. Watanabe, K. Isogai, S. Suzuki, M. Sugihara, (2002). A theoretical study of paper flutter, Journal of Fluids and Structures 16, 543-560.

[3] T.S. Balint, A.D. Lucey, (2005). Instability of a cantilevered flexible plate in viscous channel flow, Journal of Fluids and Structures, 893-912.

[4] J. C. Liao, (2007). A review of fish swimming mechanics and behaviour in altered flows. Philosophical Transactions of the Royal Society of London B: Biological Sciences, 362(1487), 1973-1993.

[5] J. A. Dunnmon, S. C. Stanton, B. P. Mann \& E. H. Dowell, (2011). Power extraction from aeroelastic limit cycle oscillations. Journal of Fluids and Structures, 27(8), 1182-1198.

[6] H. Mutsuda, J. Miyagi, Y. Doi, Y. Tanaka, H. Takao, \& Y. Sone, (2014). Flexible piezoelectric sheet for wind energy harvesting. International Journal of Energy Engineering, 4(2), 67.

[7] C. Eloy, R. Lagrange, C. Souilliez, \& L. Schouveiler, (2008). Aeroelastic instability of cantilevered flexible plates in uniform flow. Journal of Fluid Mechanics, 611, 97-106.

[8] W. Zhao; M. P. Païdoussis, L. Tang, M. Liu, \& J. Jiang, (2012). Theoretical and experimental investigations of the dynamics of cantilevered flexible plates subjected to axial flow. Journal of Sound and Vibration, 331(3), 575-587.

[9] E. Virot, X. Amandolese, \& P. Hémon, (2013). Fluttering flags: an experimental study of fluid forces. Journal of fluids and structures, 43, 385-401.

[10] R. L. Bisplinghoff., H. Ashley; \& R. L. Halfman, (2013). Aeroelasticity. Courier Corporation, chapter 3. 